\title{
Element stratification in the atmospheres of two weakly magnetic Cr-type Ap stars
}

\author{
T. Ryabchikova ${ }^{1,2}$, F. Leone ${ }^{3}$, O. Kochukhov ${ }^{2}$ and S. Bagnulo ${ }^{4}$ \\ ${ }^{1}$ Institute of Astronomy, Russian Academy of Science, 48 Pyatnitskaya str., 119017 Moscow, \\ Russia \\ email:ryabchik@inasan.rssi.ru \\ ${ }^{2}$ Institute for Astronomy, Vienna University, Türkenschanzstraße 17, A-1180 Vienna, Austria \\ ${ }^{3}$ INAF - Osservatorio Astrofisico di Catania, Via S. Sofia 78, 95123 Catania, Italy \\ ${ }^{4}$ European Southern Observatory, Casilla 19001, Santiago 19, Chile
}

\begin{abstract}
We present results of stratification analyses for $\mathrm{Mg}, \mathrm{Si}, \mathrm{Ca}, \mathrm{Cr}$ and $\mathrm{Fe}$ in the atmospheres of two Cr-type sharp-lined peculiar stars HD 133792 and HD 204411. Even in the absence of strong magnetic fields their atmospheres are stable which is confirmed by zero microturbulence. Both stars are slightly evolved, their $\log g \approx 3.5$, whereas their effective temperatures are 9200 and $8400 \mathrm{~K}$, respectively. We find a moderate $\mathrm{Cr}$ and Fe stratification. However the abundances of these elements do not fall below the solar value throughout the atmosphere. Si and $\mathrm{Ca}$ are strongly stratified, in particular in HD 133792, with large underabundances in the outer atmospheric layers. Abundance stratifications derived for weakly magnetic Ap stars are compared with the empirically determined stratification in the atmospheres of strongly magnetic Ap stars in the same temperature range.
\end{abstract}

Keywords. Stars: atmospheres, stars: abundances, stars: chemically peculiar, stars: individual; (HD 133792, HD 188041, HD 204411)

\section{Introduction}

Since the pioneering work by Babel (1992) on the theoretical and observational study of the chemical stratification in the atmosphere of the famous magnetic Ap star 53 Cam it became evident that element stratification is the common feature in most Ap stars. Ryabchikova et al. (2003) reviewed the observational evidences for abundance stratification in Bp-Ap stellar atmospheres as well as the first results of the self-consistent model atmosphere calculations which modelled diffusion processes responsible for the stratification phenomenon. Until now stratification analysis for more than 1-2 elements was performed for a few Ap stars: 53 Cam (Babel 1992), $\gamma$ Equ (Ryabchikova et al. 2002), $\beta$ CrB (Wade et al. 2003). Here we present results of the stratification analysis for three more stars: HD 133792 and HD 204411 whose magnetic field is either not detected (HD 133792) or rather weak (longitudinal field of $\approx 30 \mathrm{G}$ was measured recently by Johnson 2004), and HD 188041 which has a rather strong magnetic field.

\section{Observations}

High-resolution $(R=80000) S / N=300$ spectra of HD 133092 and HD 188041 in spectral range $\lambda \lambda 3100-10000$ were obtained using the Ultraviolet Visual Echelle Spectrograph (UVES) at the ESO VLT. An échelle spectrum of HD 204411 with $R=115000$ and $S / N=300$ in the range $\lambda \lambda 4600-7900$ was recorded with the high-resolution spectrograph SARG at the 3.55-m Telescopio Nazionale Galileo (TNG) at the Observatorio del Roque de los Muchachos (La Palma, Spain). Data were reduced in a standard way for bias 
Table 1. Atmospheric parameters of the program stars.

\begin{tabular}{ccccc}
\hline Star & $T_{\text {eff }}$ & $\log g$ & $v \sin i\left(\mathrm{~km} \mathrm{~s}^{-1}\right)$ & $B_{\mathrm{s}}, \mathrm{G}$ \\
\hline HD 133792 & 9200 & 3.6 & 0. & 1150 \\
HD 188041 & 8800 & 4.0 & 0. & 3600 \\
HD 204411 & 8400 & 3.5 & 5.4 & $<750$ \\
\hline
\end{tabular}

subtraction, flat-fielding, scattered light subtraction, order extraction, and wavelength calibration.

Table 2. Abundance distribution parameters.

\begin{tabular}{|c|c|c|c|c|c|}
\hline \multirow[t]{2}{*}{ Element } & \multicolumn{3}{|c|}{ Abundances in $\log \left(N / N_{\text {tot }}\right)$} & \multicolumn{2}{|c|}{ Abundance jump parameters in $\log \tau_{5000}$} \\
\hline & Mean & Upper atmosph. & Lower atmosph. & Position & Width \\
\hline \multicolumn{6}{|c|}{ HD 133792} \\
\hline $\mathrm{Mg}$ & -4.12 & $-2.88 \pm 0.28$ & $-4.67 \pm 0.05$ & $-2.52 \pm 0.31$ & $3.06 \pm 1.23$ \\
\hline $\mathrm{Si}$ & -4.13 & $-6.24 \pm 0.08$ & $-3.12 \pm 0.07$ & $-0.35 \pm 0.03$ & $0.49 \pm 0.13$ \\
\hline $\mathrm{Ca}$ & -6.10 & $-7.98 \pm 0.10$ & $-5.11 \pm 0.04$ & $-0.41 \pm 0.02$ & $0.01 \pm 0.77$ \\
\hline $\mathrm{Cr}$ & -3.81 & $-4.56 \pm 0.05$ & $-3.64 \pm 0.03$ & $-1.35 \pm 0.28$ & $0.10 \pm 0.64$ \\
\hline $\mathrm{Fe}$ & -3.24 & $-4.13 \pm 0.03$ & $-2.88 \pm 0.02$ & $-1.01 \pm 0.06$ & $0.11 \pm 0.01$ \\
\hline \multicolumn{6}{|c|}{ HD 204411} \\
\hline $\mathrm{Mg}$ & -4.48 & $-3.63 \pm 0.15$ & $-5.79 \pm 2.17$ & $-0.79 \pm 1.32$ & $3.11 \pm 2.77$ \\
\hline $\mathrm{Si}$ & -4.12 & $-5.74 \pm 0.23$ & $-3.76 \pm 0.02$ & $-1.00 \pm 0.14$ & $0.30 \pm 0.74$ \\
\hline $\mathrm{Ca}$ & -5.42 & $-5.72 \pm 0.04$ & $-4.24 \pm 0.02$ & $-0.72 \pm 0.19$ & $0.08 \pm 0.37$ \\
\hline $\mathrm{Cr}$ & -4.78 & $-5.10 \pm 0.09$ & $-4.63 \pm 0.16$ & $-1.03 \pm 0.38$ & $0.38 \pm 5.72$ \\
\hline $\mathrm{Fe}$ & -3.64 & $-4.59 \pm 0.03$ & $-2.73 \pm 0.04$ & $-0.66 \pm 0.06$ & $0.18 \pm 0.87$ \\
\hline \multicolumn{6}{|c|}{ HD 188041} \\
\hline $\mathrm{Si}$ & -4.00 & $-5.52 \pm 0.30$ & $-2.89 \pm 0.06$ & $-0.63 \pm 0.01$ & $0.01 \pm 0.19$ \\
\hline $\mathrm{Ca}$ & -4.82 & $-7.74 \pm 0.28$ & $-2.88 \pm 0.08$ & $-1.69 \pm 0.05$ & $2.00 \pm 0.50$ \\
\hline $\mathrm{Cr}$ & -4.07 & $-4.66 \pm 0.09$ & $-3.26 \pm 0.05$ & $-1.17 \pm 0.02$ & $0.00 \pm 0.00$ \\
\hline $\mathrm{Fe}$ & -3.80 & $-4.88 \pm 0.07$ & $-2.67 \pm 0.02$ & $-1.10 \pm 0.01$ & $0.00 \pm 0.00$ \\
\hline
\end{tabular}

\section{Atmospheric parameters, rotation and magnetic field}

To estimate model atmosphere parameters we applied the Moon \& Dworetsky (1985) calibration to the observed Strömgren photometry, taken from the catalogue of Hauck \& Mermillod (1998), and, in addition, fitted the $\mathrm{H} \alpha$ line profiles. Details of the procedure of parameter determination for HD 204411 are given in Ryabchikova et al. (2004a). For HD 188041 the optical region spectrophotometry and the $\mathrm{H} \alpha$ line profile give different gravities. Ryabchikova et al. (2004b) used $T_{\text {eff }}=8500 \mathrm{~K}, \log g=4.5$ inferred from the Strömgren photometry and optical spectrophotometry in an abundance study, whereas a lower gravity, $\log g=3.7$, provides a better fit to the $H \alpha$ line profile. A reasonable compromise between spectrophotometry and $\mathrm{H} \alpha$ is obtained with the following atmospheric parameters: $T_{\text {eff }}=8800 \mathrm{~K}, \log g=4.0$, which were used in the present study. For HD 133792 the Strömgren photometry gives $T_{\text {eff }}=8830 \mathrm{~K}$ and $\log g=3.72$. A slightly higher temperature seems to be needed for the $\mathrm{H} \alpha$ profile fit in this star. Finally, we adopted $T_{\text {eff }}=9200 \mathrm{~K}, \log g=3.6, \mathrm{M}=+0.5$ for the atmosphere of HD 133792 . For all three stars we derived the projected rotational velocities by fitting the magnetically insensitive line Fe I $\lambda 5434.52\left(g_{\text {eff }}=-0.01\right)$. No rotational broadening exceeding instrumental $\left(\approx 3.5 \mathrm{~km} \mathrm{~s}^{-1}\right)$ was needed to fit this line in HD 133792 and in HD 188792, whereas 

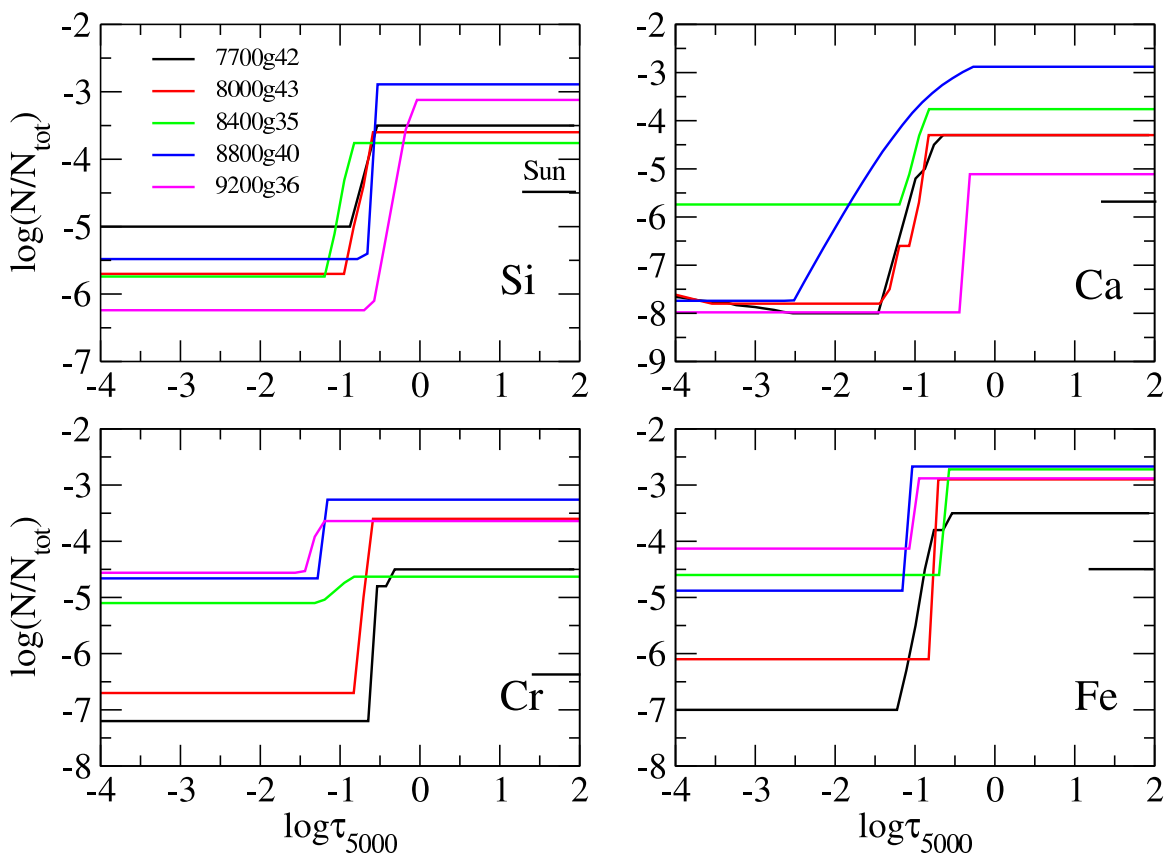

Figure 1. Element distribution in the atmospheres of Ap stars.

$v \sin i=5.4 \mathrm{~km} \mathrm{~s}^{-1}$ was derived for HD 204411. Negligible rotational velocity of HD 188041 agrees very well with the long rotational period $P=223.826 \mathrm{~d}$ derived for this star by Mikulásek et al. (2003).

Synthetic spectrum calculations with and without a magnetic field were carried out using the SYNTH3 and the SYNTHMAG_FAST_DDA codes. Atomic parameters of spectral lines were extracted from VALD database (Kupka et al. 1999) and from Raassen \& Uylings $(1998) \dagger$ in the case of CrII and Fe II lines. The mean modulus of the magnetic field $\left(B_{\mathrm{s}}\right)$ was measured directly from the splitting of magnetically sensitive Fe II $\lambda 6149.248$ in HD 188041, or was estimated from an extra broadening of the magnetically sensitive Fe I and Fe II lines in the other two stars. The adopted atmospheric parameters are given in Table 3. The Hipparcos parallaxes together with the adopted effective temperatures allow us to conclude that HD 133792 and HD 204411 have finished their life on the Main Sequence, while HD 188041 is still on the MS.

\section{Abundance stratification analysis}

Abundance analysis of HD 188041 and HD 204411 was performed by Ryabchikova et al. (2004b) and by Caliskan \& Adelman (1995) and Ryabchikova et al. (2004a), respectively. HD 204411 is known for its low abundance of the rare-earth elements (REE), which is not typical for Ap stars of Cr-type. Our preliminary analysis of the chemical composition of HD 133792 reveals moderate REE overabundances by +1.6 dex on average, which is higher by one order of magnitude than in HD 204411 and by 1.5 dex lower than in HD 188041. The average abundances of the elements for which we carried out stratification analysis are given in Table 2 .

A newly developed automatic procedure for the determination of the vertical abun-

$$
\dagger \text { ftp://ftp.wins.uva.nl/pub/orth }
$$



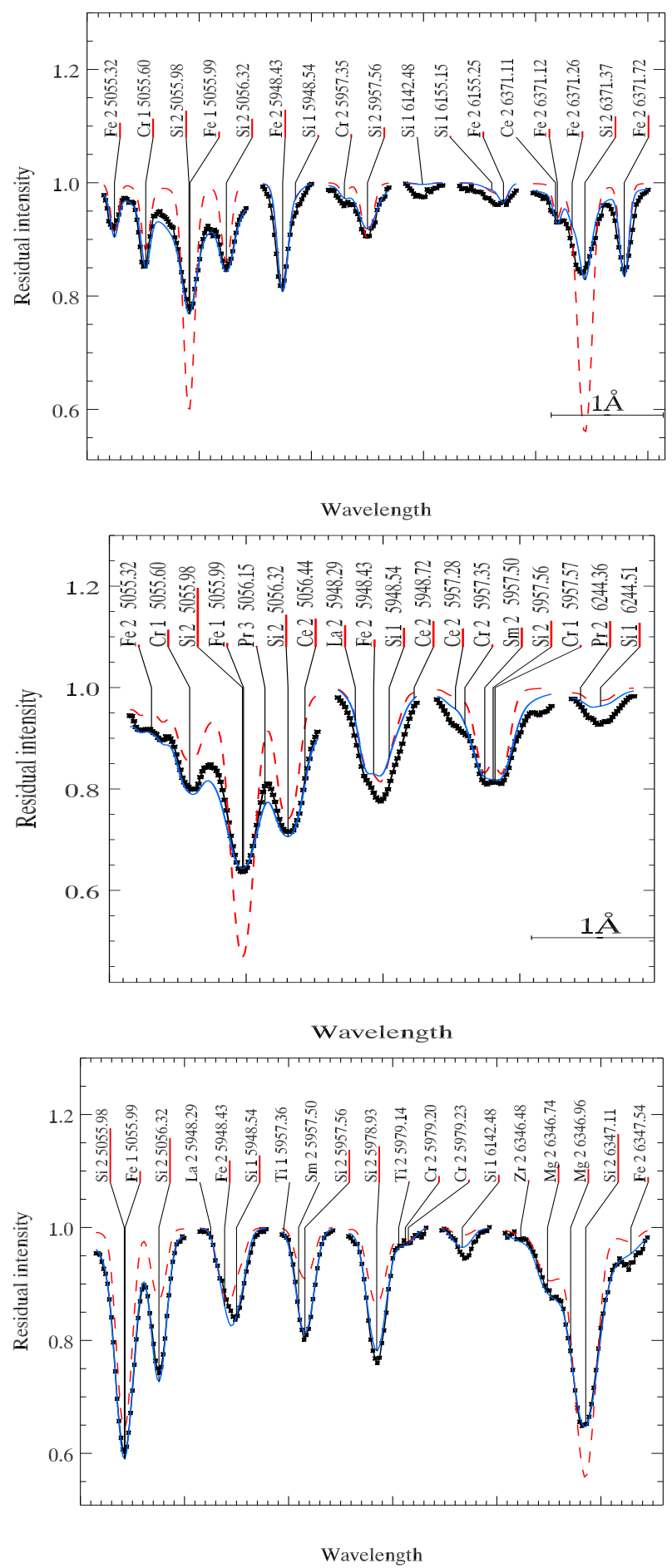

Figure 2. A comparison between the observed (dots) and calculated Si line profiles in the spectra of HD 133792 (upper left panel), HD 188041 (upper right panel) and in HD 204411 (bottom panel). Calculations for a chemically homogeneous atmospheres are shown with dashed lines, for a stratified atmospheres by solid lines. 

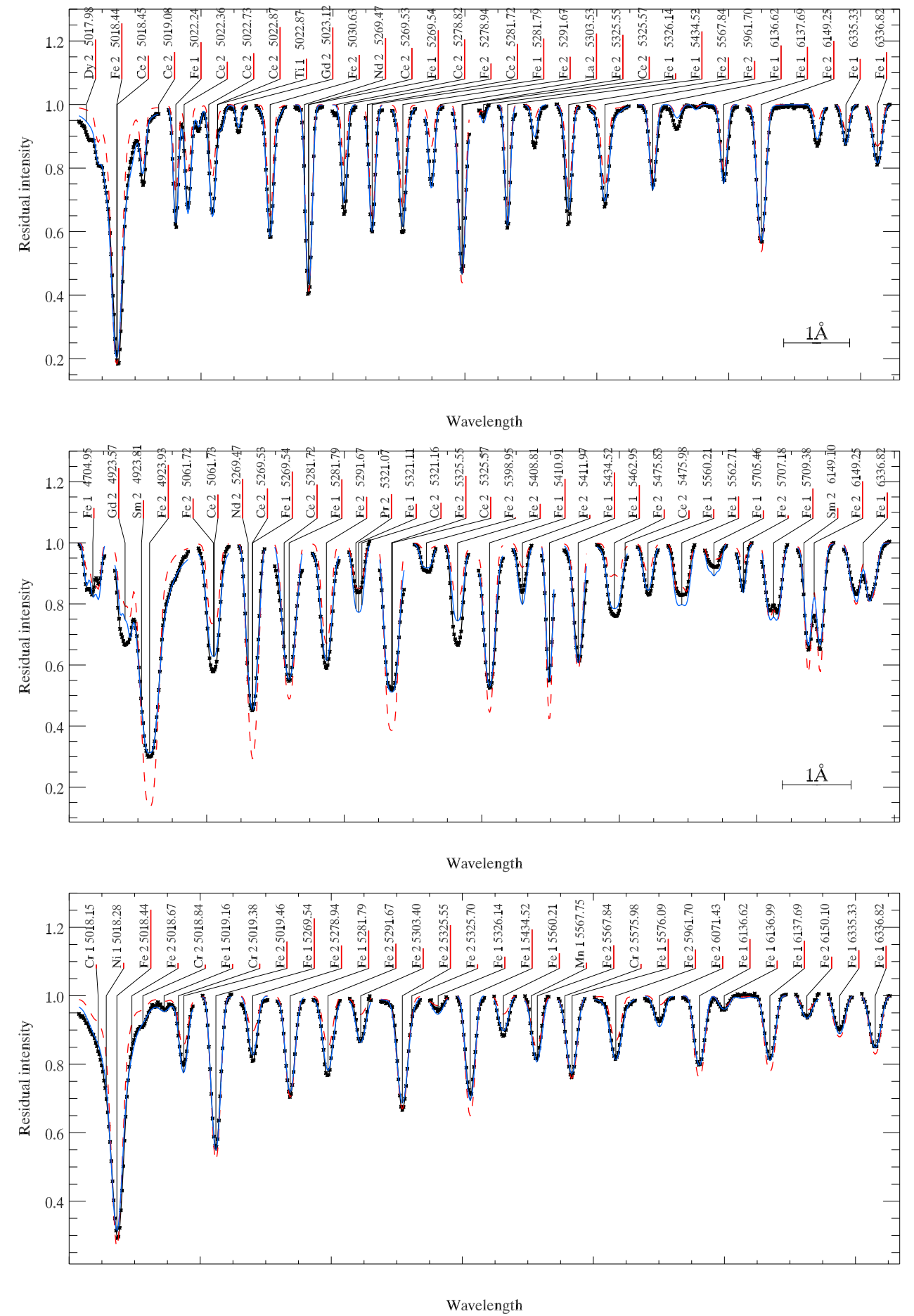

Figure 3. The same as in Fig. 2 but for Fe. The observed and computed spectra are presented for HD 133792 (top panel), for HD 188041 (middle panel) and for HD 204411 (bottom panel). 
dance gradients DDAFIT was used to derive a vertical stratification. This program is written in IDL and provides an optimization and visualization interface to the spectrum synthesis calculations with SYNTH3 in the nonmagnetic case and with SYNTHMAG_FAST_DDA in magnetic synthesis. Vertical abundance distributions are described with the four parameters: chemical abundance in the upper atmosphere, abundance in deep layers, the vertical position of the abundance step and the width of the transition region where chemical abundance changes between the two values. All four parameters can be optimized simultaneously with a least-squares fitting subroutine based on observations of an unlimited number of spectral regions, possibly using different weights in accord with the quality or relative importance of the observations of particular spectral features. The program derives one chemical stratification profile at a time, but is able to account for any number of fixed stratified abundances of, e.g., blending lines of interest.

The results of stratification analysis for the three stars are presented in Table 2 and are illustrated in Fig. 1 for Si, Ca, Cr and Fe. For comparison we show distribution of the same elements in the atmospheres of $\gamma \mathrm{Equ}\left(T_{\text {eff }}=7700 \mathrm{~K}\right)$ and $\beta \operatorname{CrB}\left(T_{\text {eff }}=8000 \mathrm{~K}\right)$. A comparison between the observed line profiles and the spectra computed with mean homogeneous and stratified element distribution is shown in Fig. 2 and Fig. 3 for Si and Fe, respectively.

\section{Conclusions}

From the spectroscopic analysis of HD 133792 and HD 204411 we found weak magnetic fields in these stars and also supported their classification as Cr-type Ap stars. Our results show that chemical stratification affects the spectra of all investigated stars in a rather large temperature range. We note that the abundance jumps occur in a relatively narrow zone of optical depths. Another important result is the behaviour of the abundance profiles with effective temperature. Although our sample is too small to make any definite conclusions, we note that with increasing effective temperature, the abundances of $\mathrm{Cr}$ and $\mathrm{Fe}$ increase in the upper atmospheric layers with a simultaneous decrease of the abundance jump itself. In the atmospheres of hotter stars the abundances of $\mathrm{Cr}$ and $\mathrm{Fe}$ may be of the same order which is supported by the diffusion predictions (see LeBlanc \& Monin 2005). We find no clear dependence of stratification on the magnetic field strength.

\section{Acknowledgements}

This work was supported by the FWF project $P$ 14984, by RFBR (grants 03-0216342, 04-02-16788) and Leading Scientific School grant 162.2003.02 to TR and by the Lise Meitner fellowship to OK (FWF project M757-N02).

\section{References}

Babel, J 1992, A $6 A$ 258, 449

Caliskan, H., \& Adelman, S.J. 1995, in: S.J. Adelman \& W.L. Wiese (eds.), Astrophysical Applications of Powerful New Databases, ASP Conf. Ser., 78, 443

Hauck, B., Mermilliod M. 1998, A\&SAS 129, 431

Johnson, N. 2004, M.Sc. Thesis, Royal Military College of Canada

Kupka, F., Piskunov, N., Ryabchikova, T.A., Stempels, H.C., Weiss, W.W. 1999, A\&AS 138, 119

LeBlanc, F., Monin, D., 2005, These Proceedings, 193

Moon, T.T., \& Dworetsky, M.M. 1985, MNRAS 217, 305

Mikulásek, Z., Žižňovský, J., Zverko, J., Polosukhina, N.S. 2003, CoSka 33, 29

Raassen, A.J.J., \& Uylings, P.H.M. 1998, A\& A 340, 300 
Ryabchikova, T., Piskunov, N., Kochukhov, O., Tsymbal, V., Mittermayer P., Weiss, W.W. 2002, A\&BA 384, 545

Ryabchikova, T., Wade, G.A., LeBlanc, F. 2003, in: N.E. Piskunov, W.W. Weiss, \& D.F. Gray (eds.), IAU Symp. 210 Modeling of Stellar Atmospheres, p. 301

Ryabchikova, T., Nesvacil, N., Weiss, W.W., Knoglinger, P., Kochukhov, O., Stütz, Ch. 2004, $A \mathscr{E} A$, (accepted)

Ryabchikova T., Leone, F., Kochukhov O. 2004, A\&A, (submitted)

Wade, G.A., LeBlanc, F., Ryabchikova, T.A., Kudryavtsev, D. 2003, in: N.E. Piskunov, W.W. Weiss, \& D.F. Gray (eds.), IAU Symp. 210 Modeling of Stellar Atmospheres, CD-D7 